\title{
Dispersion and Effective Area of Air Hole Containing Photonic Crystal Fibres
}

\author{
Nagaraju Naddi ${ }^{1}$, Eliyaz Mahammed ${ }^{2}$, K.L.Naga Ksihore ${ }^{3}$ \\ ${ }^{123}$ Department of ECE, Vardhaman College of Engineering, Hyderabad, Telangana, India
}

\begin{abstract}
Photonic Crystal Fibre (PCF) has zero dispersion at 1.55 $\mu \mathrm{m}$ wavelength compared to the standard single mode fibre $(18 \mathrm{ps} / \mathrm{hm} / \mathrm{km})$ and also sometimes negative dispersion occurs for the telecommunication wavelength range of 1.2 to $1.8 \mu \mathrm{m}$ wavelength such as the effects of air holes. The PCF design parameters on the effective index, effective mode area $\left(9.021 \mu^{2}\right.$, confinement losses $(0.0082 \mathrm{~dB} / \mathrm{m})$, and birefringence have been carefully investigated for the telecommunication wavelength range of 1.2 to $1.8 \mu \mathrm{m}$ wavelength.
\end{abstract}

Keywords: Photonic Crystal Fibres, confinement losses, Chromatic dispersion

\section{Introduction}

Optical fibre with silica air microstructures called PCF's have attracted a considerable amount of attention recently because of their unique properties that are not realized in conventional optical fibres. PCF's which are also called holey fibres or micro-structured fibres. This can be guide the light by total internal reflection between a solid core and a cladding region with multiple air holes.

The strongest wavelengths dependency of the effective refractive index and the inherently large design flexibility of the PCF's allow for a whole new range of properties. Such properties include endlessly single mode fibres and fibres with anomalous dispersion in the visible wavelengths region. PCF have applications ranging from telecommunication field to metrology, spectroscopy, microscopy, biology \&sensing. Because of its ability to confine light in hollow cores or with confinement characteristics not possible in conventional optical fibre, PCF is now finding applications in fibre optic communications fibre losses, nonlinear devices, high power transmission, highly sensitive gas sensors and other areas.

\section{Proposed Model}

The light guidance in PCF is obtained with the help of suitable array of air holes extending along the fibre propagation direction. The optical properties are related the fibre design, specifically the $\operatorname{pitch}(\Lambda)$ of the periodic array, the hole diameter(d) and the number of rings around the core. In this experiment we are designing the PCF with $\Lambda=1.6 \mu \mathrm{m}, \mathrm{d} 1 / \Lambda=0.1312, \mathrm{~d} 2 / \Lambda=0.43, \mathrm{~d} 3 / \Lambda=0.419, \mathrm{~d} 4 / \Lambda=0.92$.

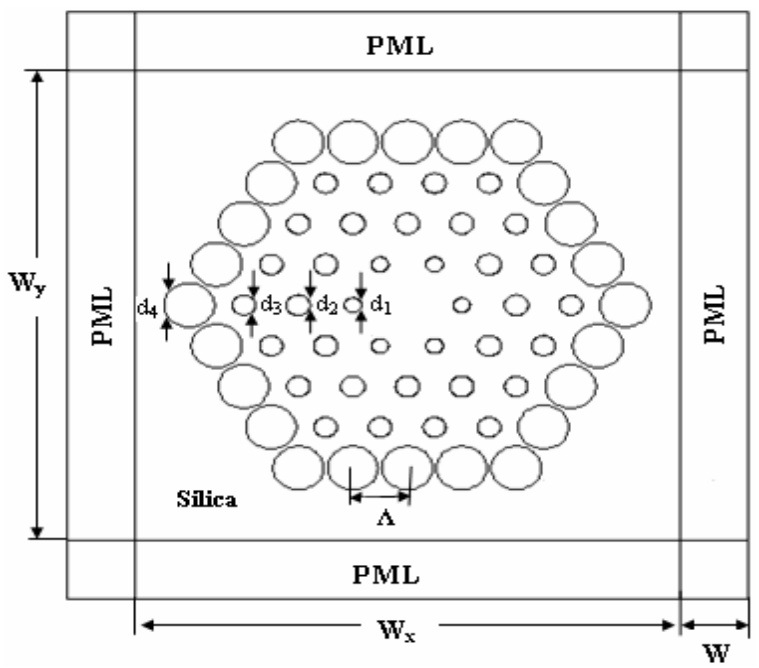

Fig 1: Schematic cross section of the PCF with four rings of 60 air holes

Figure 1 shows a schematic diagram of the PCF, consisting of four rings of arrays of air holes arranged in a silica background whose index of refraction is 1.45 . The diameter of air holes and their pitch is denoted with $\mathrm{d}$ and $\Lambda$. In solid core photonic crystal fibres, where light is confined in a higher refractive region, modified total internal reflection is exploited, which is quite similar to the guiding mechanism of standard optical fibres. 
The key factor in designing PCF is the effective mode area. The effective mode area $A_{\text {eff }}$, is related to the effective area of the core area, which is calculated by using

$$
A_{e f f}=\frac{\left(\iint E^{2} d x d y\right)^{\wedge} 2}{\iint E^{4} d x d y}
$$

The most important factor for any optical fibre technology is loss. The PCF is necessary to consider confinement loss. This is due to finite number of air holes which can be made in the fibre cross section. In solid core PCF the light is confined within a core region by the air holes. Light will move away from the core if the confinement provided by the air holes is inadequate.

$$
\begin{gathered}
\text { confinement loss }=8.686 k_{0} \operatorname{Im}\left(n_{\text {eff }}\right)(\mathrm{dB} / \mathrm{m}) \\
\text { where } k_{0}=\frac{2 \Pi}{\lambda}
\end{gathered}
$$

where $\operatorname{Im}$ is the imaginary part of the $n_{\text {eff }}$

Dispersion is defined as the signal broadening or spreading while it is propagates inside the fiber. Light waves with different wavelengths travel at different speeds inside the material. The expression for the dispersion is given below

$$
\mathrm{D}(\boldsymbol{\lambda})=-\frac{\lambda}{c} \frac{d^{2} n_{e f f}(\lambda)}{d \lambda^{2}}
$$

\section{Results And Discussion}

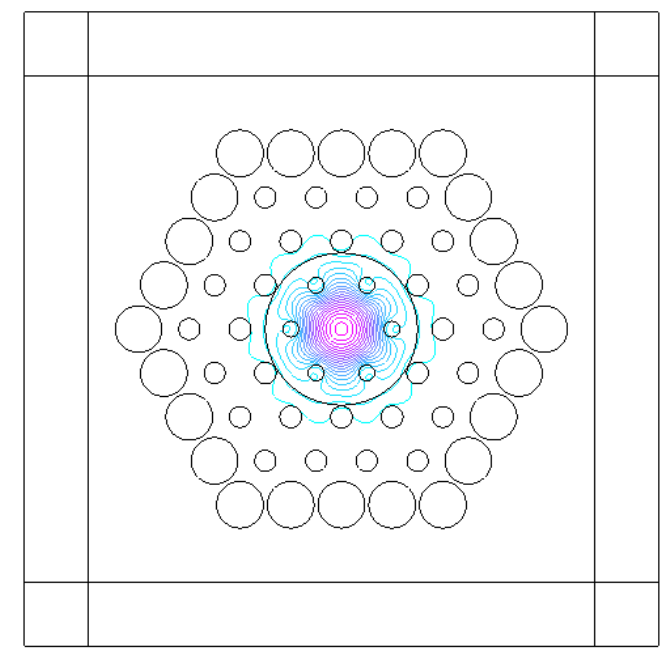

Fig 2: The field profile of the dominant $E_{x}$ component of the fundamental $H E_{11}^{x}$ mode , where $\Lambda=1.6 \mu \mathrm{m}, \mathrm{d} 1 / \Lambda$ $=0.312, \mathrm{~d} 2 / \Lambda=0.43, \mathrm{~d} 3 / \Lambda=0.419, \mathrm{~d} 4 / \Lambda=0.92$, at operating wavelength $\lambda=1.55 \mu \mathrm{m}$

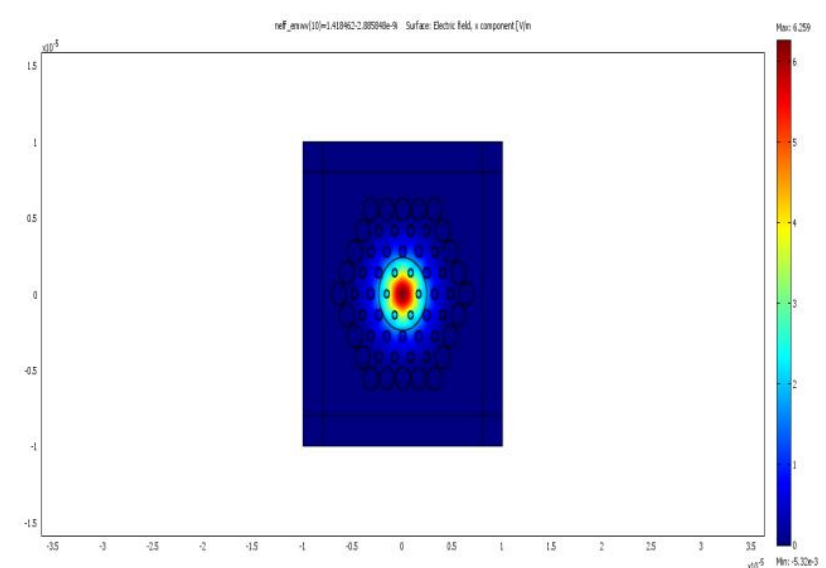

Fig 3: 2 dimensional view of the PCF 


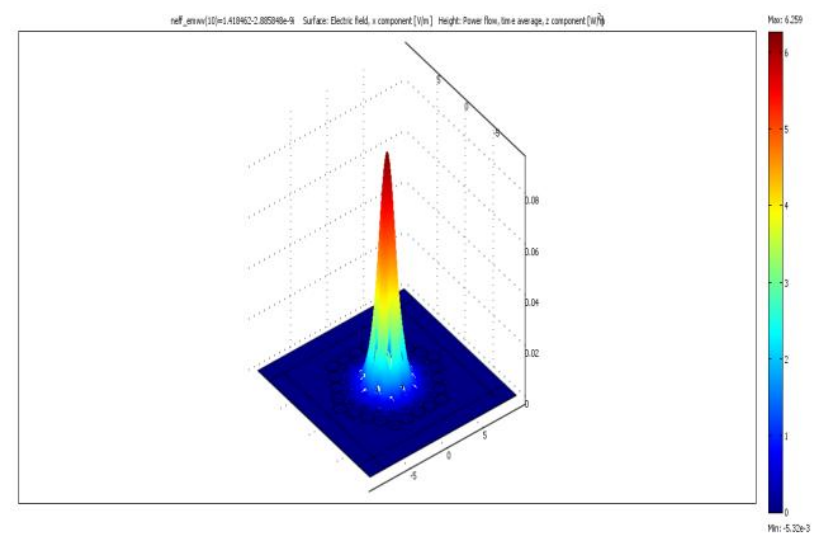

\section{Effective Mode Area}

Fig 4: 3-D view of the PCF

The variation of the effective mode area $A_{e f f}$, with the wave-length. It can be noted that $A_{e f f}$ is $9.021 \mu^{2}$ at the communication window of $1.55 \mu \mathrm{m}$. It is worth notifying that the effective area is smaller than that of conventional fibres at $1.55 \mu \mathrm{m}$ wavelength. The PCFs with nearly zero ultra flattened dispersion have relatively small effective area and would be useful for some nonlinear applications, such as super continuum generation. In the shorter operating wavelengths, mode is more confined into the core region than in the longer wavelengths.

\section{Confinement Loss}

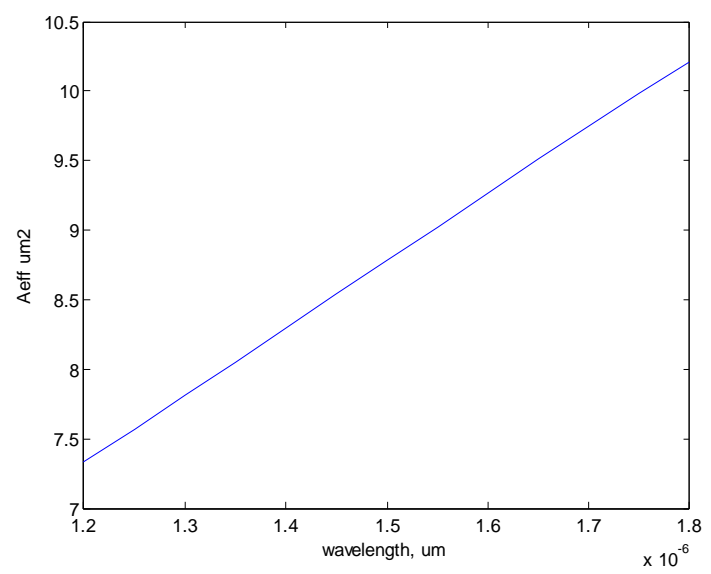

Fig5: Variation of effective mode area with wavelength

The fundamental mode is propagating in the core region. Due to a finite number of air holes, the optical mode leakage from the core region into the outer air hole region is inevitable and the confinement loss due to the extent of the cladding is taking place.

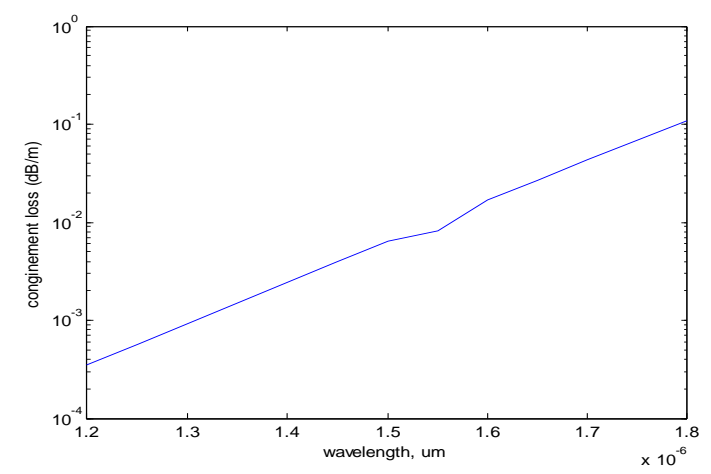

Fig6: Variation of confinement loss with the wavelength $\lambda, \Lambda=1.6 \mu \mathrm{m}, \mathrm{d} 1 / \Lambda=0.312, \mathrm{~d} 2 / \Lambda=0.43, \mathrm{~d} 3 / \Lambda=0.419$, $\mathrm{d} 4 / \Lambda=0.92, \lambda=1.55 \mu \mathrm{m}$

The confinement loss of the fundamental mode has been computed from the imaginary part of the complex effective index $n_{e f f}$ and has been from.The confinement loss decreases rapidly when the operating wavelength decreases. The confinement loss is $0.0082 \mathrm{~dB} / \mathbf{m}$ at communication wavelength $1.55 \mu \mathrm{m}$. 


\section{Dispersion}

As may be seen from the fig 7 dispersion values between the wavelengths from $1.35 \mu \mathrm{m}$ to $1.8 \mu \mathrm{m}$ can be controlled by changing the size of the air holes of the third ring. In this case almost zero dispersion has been achieved at $1.55 \mu$ mwavelength. It can also be observed that dispersion values for the wavelength from $1.5 \mu \mathrm{m}$ to $1.6 \mu \mathrm{m}$ changes between 0.4 and $-0.11 \mathrm{ps} / \mathrm{km} . \mathrm{nm}$. Negative dispersion has also been obtained around $1.55 \mu \mathrm{m}$.

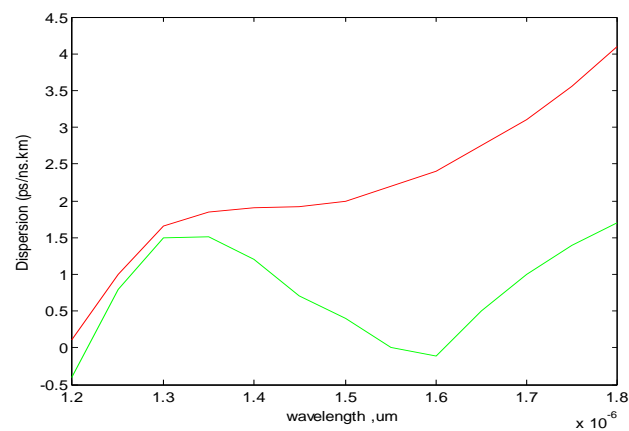

Fig 7: Variation of dispersion with wavelength where $\Lambda=1.6 \mu \mathrm{m}, \mathrm{d} 1 / \Lambda=0.312, \mathrm{~d} 2 / \Lambda=0.43, \mathrm{~d} 3 / \Lambda=0.419, \mathrm{~d} 4 /$ $\Lambda=0.92($ solid line $)$ and $\mathrm{d} 1 / \Lambda=0.312, \mathrm{~d} 2 / \Lambda=0.43, \mathrm{~d} 3 / \Lambda=0.468, \mathrm{~d} 4 / \Lambda=0.92$

\begin{tabular}{|c|c|c|c|c|c|}
\hline melengtiminometer) & red & red & ima $A$ & & confinementloss \\
\hline 12 & 1411185 & $-7 . \pi-12$ & 1411108 -781E-12 & 7.252 & -15HEOA \\
\hline 15 & 14007 & $-120=-11$ & $1430 \times 3-140=-11$ & 7. 种 & -5EECA \\
\hline 13 & 142051 & -2YE-11 & $148064-251-11$ & 78060588 & $-2 Z E-O 4$ \\
\hline 13 & 142859 & $-37 \mathbb{E}-11$ & $142503-450 E-11$ & 8004474 & $-1 \mathrm{TEEB}$ \\
\hline 14 & 14256 & $6 \pi-11$ & $1067-798=-11$ & 824202 & $-24 E-13$ \\
\hline 15 & $142 \times 04$ & $-106-10$ & $145 \times 60-140-10$ & 8570674 & $-190-B B$ \\
\hline 15 & 1424614 & $-1 \pi \mathbb{t}-10$ & 1424618 - 241E-10 & 8.3397 & 64姃-1B \\
\hline 15 & 142543 & $-23 \mathrm{k}-10$ & $142551-428 E-10$ & 902 & $\& X E-B$ \\
\hline 16 & 1051 & $-49 E-10$ & 1423.15 - G84E-10 & 92755964 & $-1 B O E-O P$ \\
\hline 16 & 142488 & 814 -10 & $14243-112=-19$ & 9517678 & $-2 \pi \in \mathbb{Q}$ \\
\hline 17 & 142069 & $-13 E-D$ & $14245-184=-19$ & 9.7Arid & 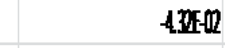 \\
\hline $1 B$ & $14 \mathrm{PH}$ & $-2 \pi \mathbf{E}=0$ & $14916-295 \mathrm{E}-\mathrm{x}$ & 99056020 & GESEL \\
\hline 18 & 1418 & -3 FE- -19 & 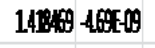 & 1020145 & -10F-11 \\
\hline
\end{tabular}

Table 1: Obtained values of effective area and confinement losses at different wavelengths

\section{Conclusion}

The effective index, the confinement loss, the effective mode area and the chromatic dispersion of the fundamental mode of this structure have been successfully investigated. Low losses with ultra-low and desired chromatic dispersion in the telecommunication wavelength are reported. It was shown that it is possible to achieve zero dispersion at $1.55 \mu \mathrm{m}$ wavelength.

\section{References}

[1] NielsAsger Mortensen and Jacob Riis Folkenberg, "Low-loss criterion and effective area considerations for photonic crystal fibres," J. Opt. A: Pure Appl. Opt., vol. 5, pp. 163-167, 2003.

[2] NielsAsger Mortensen, "Effective area of photonic crystal fibres" Optics Express, vol. 10, pp. 341-348, 2002.

[3] Kapron, F. P. (1970). "Radiation Losses in Glass Optical Waveguides". Applied Physics Letters. 17 (10): 423. Bibcode:1970ApPhL..17..423K. doi:10.1063/1.1653255.

[4] Keck, D.B. (1973). "On the ultimate lower limit of attenuation in glass optical waveguides". Applied Physics Letters. 22 (7): 307. Bibcode:1973ApPhL..22.307K. doi:10.1063/1.1654649.

[5] Kaiser P.V., Astle H.W., (1974), Bell Syst. Tech. J., 53, 1021-1039

[6] Tajima K, Zhou J, Nakajima K, Sato K (2004). "Ultralow Loss and Long Length Photonic Crystal Fibre" Journal of Light-wave Technology". Journal of Light-wave Technology. 22: 7-10. doi:10.1109/JLT.2003.822143.

[7] P. Roberts, F. Couny, H. Sabert, B. Mangan, D. Williams, L. Farr, M. Mason, A. Tomlinson, T. Birks, J. Knight, and P. St. J. Russell, "Ultimate low loss of hollow-core photonic crystal fibres," Opt. Express 13, 236-244 (2005)

[8] Canning J, Buckley E, Lyttikainen K, Ryan T (2002). "Wavelength dependent leakage in a Fresnel-based air-silica structured optical fibre". Optics Communications. 205: 95-99. Bibcode:2002 Opt Co. 2095C doi:10.1016/S0030-4018(02)01305-6 